\title{
A Novel Application of Digital Microscope for Microsurgery Training
}

Lohrasb R. Sayadi, MD ${ }^{1} \quad$ Jennifer E. Fligor, MD ${ }^{1}$ Sean Couchois, BS ${ }^{1}$ Gregory R. D. Evans, MD ${ }^{1}$ Alan D. Widgerow, MD ${ }^{1}$ Brock Lanier, MD ${ }^{1}$

${ }^{1}$ Irvine Department of Plastic Surgery, University of California, Orange, California

Address for correspondence Lohrasb Ross Sayadi, MD, Department of Plastic Surgery, The University of California, Irvine, 200 S. Manchester

J Reconstr Microsurg Open 2020;5:e32-e35. Avenue, Suite 650, Orange, CA 92868 (e-mail: rsayadi@uci.edu).

\begin{abstract}
Background Time spent under the microscope is often a limiting factor as plastic surgery residents work toward proficiency in microsurgery. This study describes and assesses a novel application of a digital microscope compatible with smart devices which can consistently and reliably magnify microsurgical fields.

Methods A digital microscope was used to display an "operating" field on a tablet device. Two junior plastic surgery residents participated in multiple training sessions. During each session, residents completed two sessions of a knot-tying task and a chicken vessel anastomosis task. The sessions were recorded on the tablet, photographed, and graded by an experienced microsurgeon utilizing three standardized microsurgery training scales (OWOMSA, OSATS, and Global scale) for evaluation. Between sessions, the residents received feedback from the experienced microsurgeon.

Results Statistically significant improvements $(p<0.05)$ in microsurgical technique were observed across 16 areas assessed by the standardized evaluation scales. Additionally, the residents' surveys suggest favorable attitudes toward the digital microscope and its value as a training device.

Conclusion Traditional operating microscopes present considerable barriers for effective microsurgical training. The digital microscope analyzed in the present study provides solutions to several of these barriers: it is economical, lightweight, portable, and can be set up by the trainee on any flat surface; photographing and recording

Keywords

- microsurgery

- digital

- education capabilities via the connected tablet device make the digital microscope setup optimized for education. Our study demonstrates measurable improvements in trainee's skills with use of-as well as favorable trainee attitudes toward-the digital microscope, which could present a valuable addition to plastic surgery education.
\end{abstract}

In Principlization of Plastic Surgery, D.R.M. writes that progress in our field requires innovations for the improvement of the standard and modification of routine to pioneer new frontiers. ${ }^{1}$ With the introduction of the triangulation method of end-to-end anastomosis in 1902, anticoagulation in 1916 and intraoperative magnification in the 1920s, the foundations were set for the first successful microvascular anastomosis using an operating microscope by Jacobson and Suarez in $1960 .^{2}$ Later technological improvements to the operating microscope, such as coaxial illumination, motorized zoom, and binocular viewing, transformed microsurgery into the form we see today. ${ }^{2}$ However, due to their specialization, these operating microscopes are costly. As time spent under the microscope is understood to relate to a received

November 7, 2019

accepted

March 20, 2020
DOI https://doi.org/

10.1055/s-0040-1710346. ISSN 2377-0813.
Copyright $\odot 2020$ by Thieme Medical

Publishers, Inc., 333 Seventh Avenue, New York, NY 10001, USA. Tel: +1(212) 760-0888.
License terms

(®) $\Theta \circledast$ 
resident's development of microsurgical proficiency, there remains a search for more accessible and cost-efficient models for training surgical residents in microsurgery.

Few studies examine more economic alternatives for microsurgical training. Several articles assess the use of a smartphone's or tablet device's camera lens as a substitute for an operating microscope in a knot-tying task. ${ }^{3,4}$ While promising, these technologies depend on the model of hardware used and are often hindered by limited magnification, focus, and image quality. Additionally, significant variability in lens quality limits standardization of said training.

In an era of ever-increasing accessibility to technology, we challenge the paradigm of microsurgical training, describing, and assessing a novel application of a digital microscope that is compatible with smartphones or tablet devices which can consistently and reliably magnify microsurgical fields.

\section{Methods}

\section{Participants}

Two junior plastic surgery residents participated in three training sessions consisting of two trials of two tasks: (1) a knot-tying task and (2) an anastomosis task.

\section{Digital Microscope Setup}

A Plugable 250× Digital USB Microscope (\$34, Plugable Technologies, Great Britain) connected to a Samsung Galaxy Tab S3 tablet device (Samsung, South Korea). The digital microscope was positioned in front of the tablet displayed over the task model ("operative field"), enabling the resident to view the field both grossly and under magnifica-

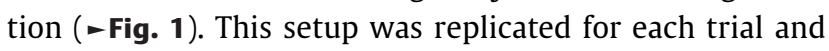
session.

\section{Latex Model}

For the knot-tying task, a latex model was constructed using a surgical latex glove draped over a wooden frame. The digital microscope was positioned over a vertical $1.0 \mathrm{~cm}$ incision in the latex (-Fig. $\mathbf{1}$ ).

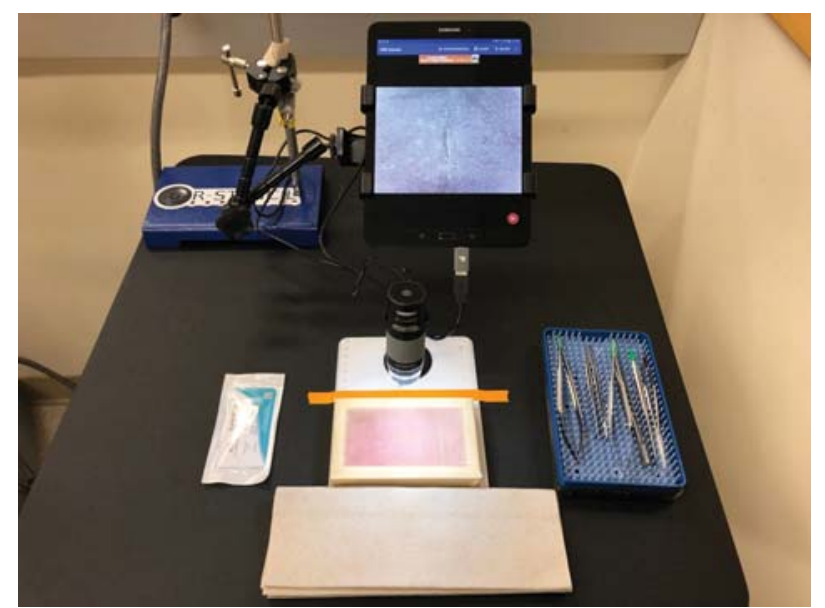

Fig. 1 Latex model with digital microscope setup.

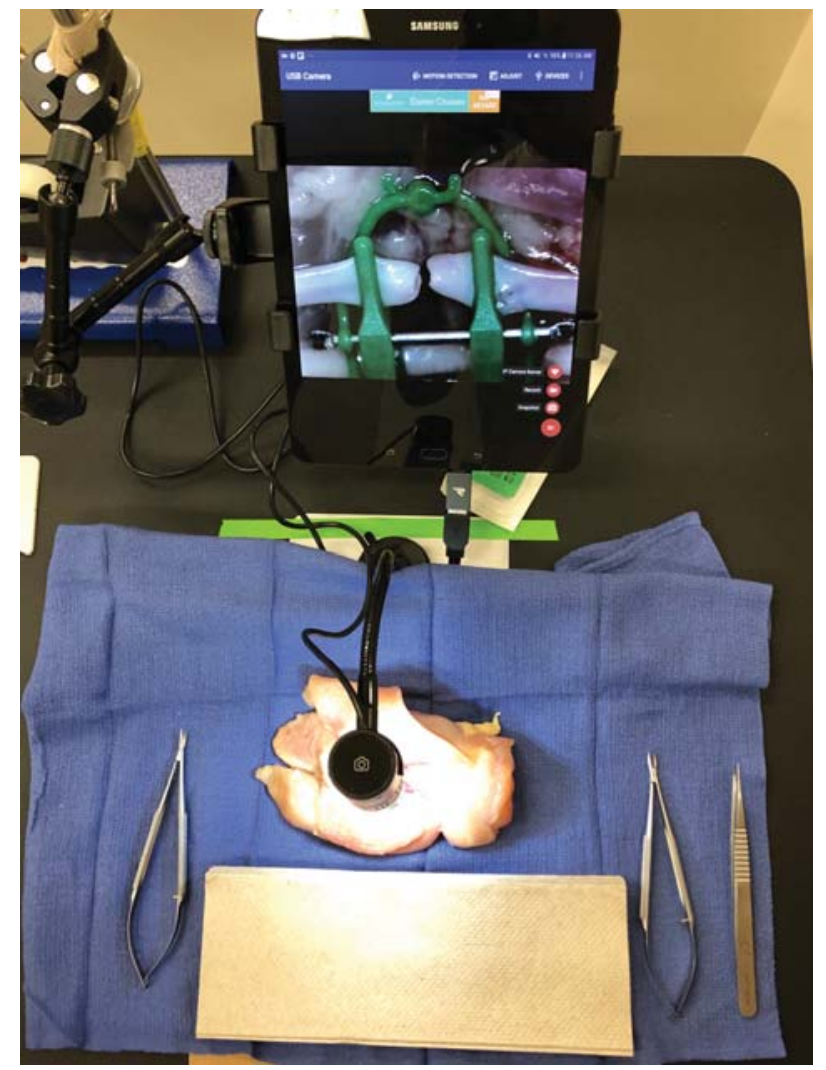

Fig. 2 Anastomosis model with digital microscope setup.

\section{Anastomosis Model}

The second task was an anastomosis model. In this model, an end-to-end microvascular anastomosis was hand sewn using interrupted 9.0 Nylon sutures in the chicken thigh model. Femoral arteries from chicken thighs were prepared, transected, and clamped using a disposable arterial vascular clip (-Fig. 2).

\section{Microsurgical Training Sessions}

Prior to the knot-tying task, the residents were asked to watch a video from Stanford's Department of Plastic and Reconstructive Surgery on the essentials of microsurgical suturing. ${ }^{5}$ Participants were then asked to repair the defect in the latex glove model with two simple interrupted sutures (-Fig. 3). The participants performed two trials of this task during each training session. A photograph was taken before and after each trial and video of the resident's performance was recorded using the tablet's screen recording option.

Prior to the anastomosis task, the residents watched a video from the University of Wisconsin's Department of Plastic Surgery, which provided technical instruction for microsurgical anastomosis. ${ }^{6}$ For the first trial, participants were asked to repair the transected femoral artery with two simple interrupted sutures. The artery was then turned 180 degrees, and a second trial was performed with the placement of an additional two simple interrupted sutures. A photograph was taken before and after anastomosis sessions and video of the resident's performance was recorded using the tablet's screen recording option (-Video 1). 


\section{Video 1}

Resident performing vessel anastomosis task. Online content including video sequences viewable at: https://www.thieme-connect.com/products/ ejournals/html/10.1055/s-0040-1710346.

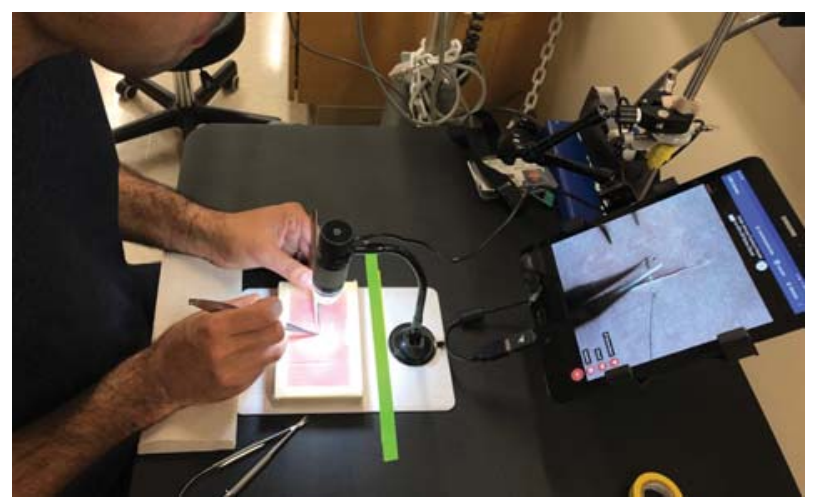

Fig. 3 Resident performing knot-tying task.

\section{Resident Evaluation}

Photographs and video recordings of the residents' performances on both the knot-tying and anastomosis tasks were evaluated and scored by a plastic surgeon with more than 20 years of microsurgery experience using three separate standardized scales for microsurgery training: The University of Western Ontario Microsurgery Skills Acquisition/Assessment (UWOMSA), ${ }^{7}$ Objective Structured Assessment of Technical Skills (OSATS)-Task Specific Score, ${ }^{8}$ and the OSATS-Global Scale. ${ }^{9-11}$ Between sessions, the trainees received feedback from the microsurgeon. After each session, the trainee completed an evaluation of the digital microscope user experience.

\section{Statistical Analysis}

All continuous variables were compared using a paired $t$-test with a significance level of $p<0.05$. Statistical comparisons were made between sessions ( $\mathrm{S} 1, \mathrm{~S} 2$, and S3).

\section{Results}

Statistically significant improvements in scores were seen in several measures of resident microsurgical technique over the course of the three-session curriculum.

For the knot-tying task, participants demonstrated significant improvements on parameters assessed by each of the three scales used in evaluation. Significant improvements on the UWOMSA scale were found in (1) quality of knot from S1 to S3 $(p<0.05)$ and $(2)$ total score from S1 to S3 $(p<0.05)$. Significant improvement on the OSATS-Task Specific Score was found for total score from S1 to S2 $(p<0.05)$ and from S1 to S3 $(p<0.05)$. Significant improvement on the OSATSGlobal Score was demonstrated in (1) instrument handling from S1 to S3 ( $p<0.01)$, and (2) suture handling from S1 to S3 $(p<0.01)$, the (3) quality of final product from S1 to S2 $(p<0.05)$ and from S1 to S3 $(p<0.01)$, and the (4) total score from S1 to S3 $(p<0.001)$.

For the anastomosis task, the UWOMSA scale revealed statistically significant improvements in (1) suturing technique from $S 1$ to $S 2(p<0.05)$ and from $S 1$ to $S 3(p<0.0001)$, (2) quality of final product from S1 to $S 2(p<0.05)$, and in (3) total score from S1 to S2 $(p<0.05)$. The OSATS-Task Specific Score demonstrated significant improvement in total score from S1 to S2 $(p<0.01)$ and from S1 to S3 $(p<0.01)$. Significant improvement on the OSATS-Global Scale, (1) respect for tissue significantly improved from S1 to S2 $(p<0.05)$, as well as (2) time and motion from S1 to S2 $(p<0.0001)$ and from S1 to S3 $(p<0.01)$, (3) instrument handling from S1 to S3 $(p<0.05)$, (4) quality of final product from S1 to S2 $(p<0.01)$ and from $S 1$ to S3 $(p<0.01)$, and the (5) total score from S1 to S2 $(p<0.01)$ and from S1 to S3 $(p<0.05)$.

According to the surveys completed by the participants after each session, the residents had a $90 \%$ likelihood of: (1) using the device for practicing microsurgery at home (2) recommending the device to other colleagues and (3) endorsing the utility of the device for improving their microsurgical skill set.

\section{Discussion}

An aspiring reconstructive surgeon must devote more hours to the practice of microsurgery to develop proficiency than mastery of microsurgical skills. Unfortunately, the traditional operating microscope presents considerable barriers to training, including cost, portability, and availability of the instrumentation.

The digital microscope described and evaluated in the present study provides solutions to these barriers. Standard operating microscopes cost $\$ 10,000$ to $\$ 300,000$, whereas the digital microscope costs around $\$ 34$ and can be connected to a resident's smartphone or tablet device. This feature could prove particularly valuable for applications in resource-poor settings. In addition, the portability of the device frees the trainee from a microsurgery laboratory, allowing for practice virtually anywhere. The setup described is forgiving for the trainee in first becoming familiar with microsurgical instruments and sutures, allowing them to view the "operating" field both grossly and under magnification on the tablet screen. The setup enables photographs and video recordings, which can then be reviewed by the resident or an instructor. Our results demonstrate that the digital microscope is a viable setup for microsurgical training associated with statistically significant improvements in both knot tying and anastomosis related to suturing technique, time and motion, and instrument handling and quality of the final product in only three practice sessions.

This study and the digital microscope setup are not without limitations. Trainees reported limited depth perception due to the monocular nature of the digital microscope lens and instances of technological lag interfering with their training. We believe the former may be improved with an additional digital lens stitched together in virtual reality to yield superior depth perception. While we believe the improvements in 
resident microsurgical technique acquired with digital microscope training will translate to improved prowess under the operative microscope, no formal assessment of this was made.

Our study demonstrates measurable improvement in trainees' skills with the use of the digital microscope, as well as favorable trainee attitudes toward the setup. The digital microscope setup could represent a valuable addition to plastic surgery education.

\section{Note}

All the principles outlined in the Helsinki Declaration of 1975 , as revised in 2000 , have been followed in all the experiments involving human subjects during the current study.

\section{Conflict of Interest}

None declared.

\section{References}

1 Millard DR. Principlization of Plastic Surgery. 1st ed. Boston, MA: Little, Brown; 1986

2 Tamai S. History of microsurgery. Plast Reconstr Surg 2009;124 (06):e282-e294

3 Karakawa R, Yoshimatsu H, Yano T, Sawaizumi M. Microsurgery training using Apple iPad Pro. Microsurgery 2018;38(08):926-927
4 Capkin S, Cavit A, Kaleli T. Microsurgery training with smartphone. Handchirurgie, mikrochirurgie, plastische chirurgie: organ der deutschsprachigen arbeitsgemeinschaft fur handchirurgie. Organ der Deutschsprachigen Arbeitsgemeinschaft fur Mikrochirurgie der Peripheren Nerven und Gefasse 2018;50(06):443-445

5 Microsurgery essentials: suturing [video online]. Available at: https://plasticsurgery.stanford.edu/education/microsurgery/suturing.html. Accessed April 2, 2020

6 Blue "blood" gives residents innovative microsurgery training. https://www.surgery.wisc.edu/2018/12/13/blue-blood-gives-residents-innovative-microsurgery-training/. Accessed April 2, 2020

7 Temple CL, Ross DC. A new, validated instrument to evaluate competency in microsurgery: the University of Western Ontario Microsurgical Skills Acquisition/Assessment instrument [outcomes article]. Plast Reconstr Surg 2011;127(01):215-222

8 Martin JA, Regehr G, Reznick R, et al. Objective structured assessment of technical skill (OSATS) for surgical residents. $\mathrm{Br} \mathrm{J}$ Surg 1997;84(02):273-278

9 Regehr G, MacRae H, Reznick RK, Szalay D. Comparing the psychometric properties of checklists and global rating scales for assessing performance on an OSCE-format examination. Acad Med 1998;73(09):993-997

10 Grober ED, Hamstra SJ, Wanzel KR, et al. Validation of novel and objective measures of microsurgical skill: hand-motion analysis and stereoscopic visual acuity. Microsurgery 2003;23 (04):317-322

11 Reznick R, Regehr G, MacRae H, Martin J, McCulloch W. Testing technical skill via an innovative "bench station" examination. Am J Surg 1997;173(03):226-230 